\title{
Equilibria in multi-player multi-outcome infinite sequential games
}

\author{
Stéphane Le Roux \\ Department of Mathematics, Technische Universität Darmstadt ${ }^{1}$, Germany \\ leroux@lsv.fr \\ Arno Pauly \\ Department of Computer Science, Swansea University, UK \\ a.m.pauly@swansea.ac.uk
}

\begin{abstract}
We investigate the existence of various types of equilibria (Nash, subgame perfect, Pareto-optimal, secure) in multi-player multi-outcome infinite sequential games. Our results are transfer theorems: Assuming determinacy for a class of simple two-player win/lose games, we obtain existence results about equilibria in the associated multi-player multi-outcome games.

Keywords: perfect information, Borel measurable, determinacy, Nash, subgame-perfect, Pareto optimal, secure equilibrium

2010 MSC: 91A13, 91A18, 03E15

\section{Introduction}

We investigate the existence of various kinds of equilibria in multi-outcome multi-player infinite sequential games. Our results are transfer theorems that construct such equilibria from winning strategies in derived, simpler two-player 5 win/lose games. Together with Borel determinacy [1], or even assuming the axioms of projective determinacy $(\mathrm{PD})$ or determinacy $(\mathrm{AD})$, we can conclude the existence of such equilibria.
\end{abstract}

\footnotetext{
${ }^{1}$ Le Roux has since moved to the Laboratoire Spécification et Vérification at ENS ParisSaclay.
}

Preprint submitted to Information $\&$ Computation

December 9, 2018 
Rather than working with real-valued payoff functions, as common in this area, we use strict weak orders as preferences. This is a proper generalization, and better behaved regarding constructions such as taking lexicographic products (cf. [2, Proposition 43]). In Section 5 we mention corollaries about games with real-valued payoff functions, which we obtain from our main theorems. For contrast we also mention known results in this area.

Section 3 is an extension of the conference paper 3], and improves upon results in 4. There we investigate subgame-perfect equilibria in antagonistic games as well as Nash equilibria in a general setting. In Section 4 we then look at Pareto-optimal equilibria (Definition 7) and secure equilibria (Definition 9).

The research programme this article contributes to reunites two mostly separate developments in the study of games. On the one hand, the first development ${ }_{20}$ is the investigation of variations on solution concepts for games, and of different formalizations of the preferences of the players, which primarily happened inside game theory proper. On the other hand, the study of infinite sequential games has a long history in logic. Many variations on the rules of games have been studied, albeit mostly restricted to zero-sum games with two players and two outcomes. The celebrated core result here is Borel determinacy (Martin [1]): In a two-player win/lose game where the winning set is Borel, one of the two players has a winning strategy.

A similar synthesis of the approaches is found in e.g. [5, 6, 7, 8]. In this area, typically results either are given only for low complexity settings (such as semicontinuous payoff functions), or they reduce the case at hand to Borel determinacy (which is how we proceed here). There are various existence theorems for equilibria known, and various counterexamples. The precise requirements needed to necessitate the existence of certain equilibria are generally unknown. In some cases, we are able to give exact classifications (Theorems 16, 21), in 35 others, we are merely pushing forward the boundary of the known. We discuss the state of the art some more in Subsection 3.1 and at the end of Section 5

Our main interest in pursuing this theme is to better understand the interplay between features of the preferences of players and of types of equilibria. 
Contrasting Theorems 16 and 21, for example, shows that for Nash equilibria to exist, the fundamental obstruction is (informally spoken) repeated improvement (in a narrow sense) can still leave a player off worse in the limit; while for pareto-optimal equilibria the obstruction is the same pattern in the preferences that also pertains to subgame-perfect equilibria in other settings.

A similar move from two-player win/lose games to multi-player multi-outcome games is occurring in games used for verification and synthesis in theoretical computer science (e.g. 9] for multi-outcome, 6] for multi-player). Here the winning conditions (respectively preferences) are much more structured than just being Borel: a common assumption would be $\omega$-regularity. In turn, the desired winning strategies (respectively equilibria) would be realized by finite automata. Transfer results in the same spirit as in the present paper are implicitly present in [10, and very explicitly in [2] (which was inspired by [3], the precursor of this article).

\section{Background}

In our most abstract definition, a game is a tuple $\left\langle A,\left(S_{a}\right)_{a \in A},\left(\prec_{a}\right)_{a \in A}\right\rangle$ consisting of a non-empty set $A$ of agents or players, for each agent $a \in A$ a non-empty set $S_{a}$ of strategies, and for each agent $a \in A$ a preference relation $\prec_{a} \subseteq\left(\prod_{a \in A} S_{a}\right) \times\left(\prod_{a \in A} S_{a}\right)$. The generic setting suffices to introduce the notion of a Nash equilibrium: a strategy profile $\sigma \in\left(\prod_{a \in A} S_{a}\right)$ is called a Nash equilibrium, if for every agent $a \in A$ and every strategy $s_{a} \in S_{a}$ we find ${ }_{60} \neg\left(\sigma \prec{ }_{a} \sigma_{a \mapsto s_{a}}\right)$, where $\sigma_{a \mapsto s_{a}}$ is defined by $\sigma_{a \mapsto s_{a}}(b)=\sigma(b)$ for $b \in A \backslash\{a\}$ and $\sigma_{a \mapsto s_{a}}(a)=s_{a}$. In words, no agent prefers over a Nash equilibrium some other situation that only differs in her choice of strategy.

We will consider games where strategy spaces and preferences are derived objects from more structured variants of games. One such variant is the infinite sequential game:

Definition 1 (Infinite sequential game, cf. [4, Definition 1.1]). An infinite sequential game is an object $\left\langle A, C, d, O, v,\left(\prec_{a}\right)_{a \in A}\right\rangle$ complying with the following. 
1. $A$ is a non-empty set (of agents).

2. $C$ is a non-empty set (of choices).

3. $d: C^{*} \rightarrow A$ (assigns a decision maker to each stage of the game).

4. $O$ is a non-empty set (of possible outcomes of the game).

5. $v: C^{\omega} \rightarrow O$ (assigns outcomes to infinite sequences of choices).

6 . Each $\prec_{a}$ is a binary relation over $O$ (modeling the preference of agent $a$ ).

Here $C^{*}$ denotes the set of finite sequences over $C$, and $C^{\omega}$ the set of infinite 75 sequences over $C$.

The intuition behind the definition is that agents take turns to make a choice. Whose turn it is depends on the past choices via the function $d$. Over time, the agents thus jointly generate some infinite sequence, which is mapped by $v$ to the outcome of the game. Note that using a single set of actions $C$ for each step just simplifies the notation, a generalization to varying action sets is straightforward.

The infinite sequential games can be seen as abstract games: the agents remain the agents and the strategies of agent $a$ are the functions $s_{a}: d^{-1}(\{a\}) \rightarrow$ $C$. We can then safely regard a strategy profile as a function $\sigma: C^{*} \rightarrow C$ whose induced play is defined below, where for an infinite sequence $p \in C^{\omega}$ we let $p_{n}$ be its $n$-th value, and $p_{\leq n}=p_{<n+1} \in C^{*}$ be its finite prefix of length $n$.

Definition 2 (Induced play and outcome, cf. 4, Definition 1.3]). Let $s: C^{*} \rightarrow$ $C$ be a strategy profile. The play $p=p^{\gamma}(s) \in C^{\omega}$ induced by starting at $\gamma \in C^{*}$ is defined inductively through its prefixes: $p_{n}=\gamma_{n}$ for $n \leq|\gamma|$ and 9o $p_{n}:=s\left(p_{<n}\right)$ for $n>|\gamma|$. Also, $v \circ p^{\gamma}(s)$ is the outcome induced by starting at $\gamma$. The play (resp. outcome) induced by $s$ is the play (resp. outcome) induced by $s$ starting at $\varepsilon$.

In the usual way to regard an infinite sequential game as a special abstract game, an agent prefers a strategy profile $\sigma$ to $\sigma^{\prime}$, iff he prefers the outcome induced by $\sigma$ to the outcome induced by $\sigma^{\prime}$. And indeed we shall call a strategy profile of an infinite sequential game a Nash equilibrium, iff it is a Nash 
equilibrium with these preferences. In a certain notation overload, we will in particular use the same symbols for the preferences over strategy profiles and the preferences over outcomes.

The above translation of sequential games into abstract games yields the standard concept of Nash equilibrium for sequential games. However, this concept does not capture rationality as much as desirable: players may indeed use empty threats, i.e. declarations they would play in a certain way from a position onwards, even if it would be against their own interests once the position is reached. As long as the empty threats keep other players from moving to that position, they may be used in a Nash equilibrium nonetheless. This lack of rationality can be fixed by considering the concept of subgame perfect equilibrium [11. It is usually seen as an alternative concept to (actually a restriction of) Nash equilibrium, but Definition 3 defines it simply by considering an alternative translation of the preferences from sequential games to abstract games. (Similar remarks were made in [12, Lemma 144 in Section 7.2.3, Section 7.3.2].)

Definition 3. Given an infinite sequential game $\left\langle A, C, d, O, v,\left(\prec_{a}\right)_{a \in A}\right\rangle$, let the subgame perfect preferences $2^{2} \prec_{a}^{s g p} \subseteq C^{C^{*}} \times C^{C^{*}}$ be defined by $\sigma \prec_{a}^{s g p} \sigma^{\prime}$ iff $\exists \gamma \in C^{*}$ such that $p^{\gamma}(\sigma) \prec_{a} p^{\gamma}(\sigma)$.

115 The subgame perfect equilibria of $\left\langle A, C, d, O, v,\left(\prec_{a}\right)_{a \in A}\right\rangle$ are the Nash equilibria of $\left\langle A,\left(C^{d^{-1}(\{a\})}\right)_{a \in A},\left(\prec_{a}^{s g p}\right)_{a \in A}\right\rangle$.

We consider a further variant, namely the infinite sequential games with realvalued payoffs, which can (but do not have to) be understood as a special case of infinite sequential games.

${ }_{120}$ Definition 4. An infinite sequential game with real-valued payoffs is a tuple $\left\langle A, C, d,\left(f_{a}\right)_{a \in \mathbb{N}}\right\rangle$ where $A, C, d$ are as above, and $f_{a}: C^{\omega} \rightarrow \mathbb{R}$ is the payoff function of player $a$.

\footnotetext{
${ }^{2}$ Note that the translation of preferences in the following definition does not preserve acyclicity. Preservation could be ensured, e.g., by giving the nodes a linear "priority" order, in a lexicographic fashion. This, however, would complicate the definition against little benefit for the point that we want to make.
} 
Such a game can be identified with the infinite sequential game

$$
\left\langle A, C, d, \mathbb{R}^{A}, v,\left(\prec_{a}\right)_{a \in A}\right\rangle
$$

where $v(p)=\left(f_{a}(p)\right)_{a \in A}$ and for $x, y \in \mathbb{R}^{A}$, we set $x \prec_{a} y$ iff $x_{a}<y_{a}$.

As with the introduction of the subgame perfect equilibria, we can consider infinite sequential games with real-valued payoffs as infinite sequential games in several ways. One way gives rise to another commonly studied equilibrium concept, namely $\varepsilon$-Nash equilibria. Given some $\varepsilon>0$, we define the relation $\prec_{a}^{\varepsilon} \subseteq \mathbb{R}^{A} \times \mathbb{R}^{A}$ by $x \prec_{a}^{\varepsilon} y$ iff $y_{a}-x_{a}>\varepsilon$. Using $\prec_{a}^{\varepsilon}$ in place of $\prec_{a}$ in Definition 4 then provides the notion of $\varepsilon$-Nash equilibrium. Furthermore, by combining the two alternative ways (the one for SPE and the one for $\varepsilon-\mathrm{NE}$ ) to translate from infinite sequential games to abstract games, we also obtain $\varepsilon$-subgame perfect equilibria.

For infinite sequential games with real-valued payoffs, every Nash equilibrium (w.r.t. the standard preferences) is an $\varepsilon$-Nash equilibrium; and every subgame perfect equilibrium is an $\varepsilon$-subgame perfect equilibrium. Moreover, every subgame perfect equilibrium is a Nash equilibrium, and in particular, every $\varepsilon$-subgame perfect equilibrium is an $\varepsilon$-Nash equilibrium.

We use antagonistic game to refer to two-player games with preferences satisfying $\prec_{a}=\prec_{b}^{-1}$, where $x \prec^{-1} y \Leftrightarrow y \prec x$.

Definition 5 recalls a few more notions that are only tangentially related to the formulation of our results, but that do show up in the proofs.

Definition 5. A two-player win/lose game is a tuple $\langle C, D, W\rangle$ with $D \subseteq C^{*}$ and $W \subseteq C^{\omega}$. It corresponds to the infinite sequential game $\langle\{a, b\}, C, d,\{0,1\}, v,\{<$ ,$\left.\left.<^{-1}\right\}\right\rangle$ where $d$ is defined via $d^{-1}(\{a\})=D$ and $v$ is defined via $v^{-1}(\{1\})=W$.

We extend the notion of the induced play. Given some partial function $s: \subseteq C^{*} \rightarrow C$, we define the consistency set $P(s) \subseteq C^{\omega}$ by:

$$
P(s)=\left\{p(\sigma)\left|\sigma: C^{*} \rightarrow C \wedge \sigma\right|_{\operatorname{dom}(s)}=s\right\}
$$

Strict weak orders

A strict weak order is a strict partial order whose complement is transitive. 
Definition 6 (Strict weak order). A relation $\prec$ is called a strict weak order if it satisfies:

$$
\begin{aligned}
& \forall x, \quad \neg(x \prec x) \\
& \forall x, y, z, \quad x \prec y \wedge y \prec z \Rightarrow x \prec z \\
& \forall x, y, z, \quad \neg(x \prec y) \wedge \neg(y \prec z) \Rightarrow \neg(x \prec z)
\end{aligned}
$$

Said otherwise, a strict weak order is a partial order whose non-comparability relation is an equivalence relation. We write $x \sim y$ to denote $\neg(x \prec y) \wedge \neg(y \prec x)$ and $x \precsim y$ to denote $x \sim y \vee x \prec y$. An important property is that the quotient $\prec / \sim$ is a strict linear order (over the equivalence classes of $\sim$ ).

The usual preferences induced by real-valued payoff functions are strict weak orders. However, strict weak orders are more general than real-valued payoff functions (for an example, see e.g. [2]). Many results in this article, more specifically their proof techniques, rely on the preferences being strict weak orders.

Given a strict weak order $\prec$ over a set $O$, a $\precsim$-terminal interval is a set $I$ satisfying the formula $\forall x, y \in O, x \precsim y \wedge x \in I \Rightarrow y \in I$. Initial intervals are defined likewise, and an interval is extremal if it is terminal or initial.

\section{Pareto-optimality}

Pareto-optimality provides a notion of social desirability in game theory, and can be used either to pick particularly relevant equilibria, or to investigate whether the strategic interaction is costly in some sens $\mathrm{H}^{3}$. In this article we study the latter. The fundamental idea is that a Pareto-optimal outcome cannot be improved for someone without being worsened for someone else. Pareto-optimality is often only defined for real-valued payoff tuples, but the same, syntactically, definition also makes sense for strict weak orders, and more generally for strict partial orders.

Definition 7. An outcome is realizable in some game, if it is assigned to some sequence of choices. We call an outcome o Pareto-optimal, if there is no other

\footnotetext{
${ }^{3}$ Similar to the (quantitative) price of stability, see [13.
} 
realizable outcome $q$ such that for some player $a$ we find $o \prec_{a} q$ and for no player $b$ we have $q \prec_{b} O$.

We shall call a Nash equilibrium Pareto-optimal iff it induces a Paretooptimal outcome. For this notion of Pareto-optimality, there are games with NE but without Pareto-optimal NE:

Example 8. Let $z \prec_{a} y \prec_{a} x$ and $x \prec_{b} z \prec_{b} y$. The game below has only one Nash equilibrium, which yields outcome $z$. However, both players would prefer the realizable outcome $y$. Thus, the unique Nash equilibrium is not Paretooptimal.

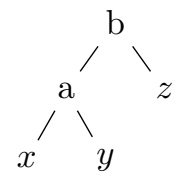

We characterize sequential games admitting Pareto-optimal Nash equilibria in Subsection 4.1 .

Secure equilibria

Secure equilibria were introduced in connection with model checking [14, for two-player games whose outcomes are in $\{0,1\}^{2}$. They were then generalized into quantitative secure equilibria [15], for two-player games whose outcomes are in $\mathbb{R}^{2}$. They were again generalized in [16, for $n$-player games with outcomes in $\mathbb{R}^{n}$. The (quantitative) secure equilibria of a game are the Nash equilibria of another game obtained by changing the usual preference of each player into a malevolent preference: instead of just trying to maximize her own payoff, she tries primarily to do so and, in case of ties, to minimize the opponents' payoff. Here we generalize further the secure equilibria, for multi-player games with strict weak order preferences.

Definition 9. The secure equilibria of a game $\left\langle A, C, d, O, v,\left(\prec_{a}\right)_{a \in A}\right\rangle$ are the Nash equilibria of the derived game $\left\langle A, C, d, O, v,\left(\prec_{a}^{\prime}\right)_{a \in A}\right\rangle$, where for all $a \in A$ we set $x \prec_{a}^{\prime} y$ if either $x \prec_{a} y$ or the following conditions are all satisfied: 
- $\exists b \in A, y \prec_{b} x$

- $\forall b \in A, y \precsim_{b} x$

We discuss the existence of secure equilibria in Subsection 4.2

Descriptive set theory

We mention certain concepts from descriptive set theory. The precise definitions are not required to follow the presentation here, but we include them for completeness. A pointclass is just a set of subset of $C^{\omega}$. A pointclass is called determined if for every element $W$ of the pointclass the two-player win/lose games with $W$ as winning set are determined. Pointclasses of particular relevance for us are the Borel and the quasi-Borel sets. We start with $\left\{w C^{\omega} \mid w \in C^{*}\right\}$ and close it under union to obtain the open sets. Closing the open sets under countable union and complement yields the Borel sets. Adding in closure under union of open-separated families gives the quasi-Borel sets, where a family $\left(A_{i}\right)_{i \in I}$ of sets is open-separated, if there is a disjoint family of open sets $\left(O_{i}\right)_{i \in I}$ with $A_{i} \subseteq O_{i}$. If $C$ is countable, the Borel sets and quasi-Borel sets coincide and are just the closure of the class $\left\{w C^{\omega} \mid w \in C^{*}\right\}$ under countable union and complement. It is a folklore generalization of Martin's theorem that the quasi-Borel sets are determined, cf. [17]. We also mention $\Delta_{2}^{0}$-sets, these are those that are simultaneously obtainable from open sets by taking countable intersections and from complements of open sets by countable union.

\section{Nash and subgame-perfect equilibria}

\subsection{What is known}

Games with finite action sets and continuous payoff functions are known to have subgame-perfect equilibria, as originally shown by Fudenberg and Levin [18. The topology used on the strategies here is just the product topology derived from the discrete topology on the action sets. Over the years, many 
improvements and extensions of the original setting have been obtained, for example with more general action sets [19] or with partial information in dynamical games 20]. The fundamental continuity of the payoff functions has not been relaxed however, and we will show below why.

Approximate equilibria can be obtained provided that the payoff functions are Borel-measurable and bounded. This is a classic result, and a proof can be found e.g. at [21, Page 97]. It is also a direct corollary of our Theorem 15. Theorem 15 shows what the sufficient and necessary criteria are for the fundamental idea behind the classical result to go through. Approximate subgame-perfect equilibria exist in two-player antagonistic games. This is again a classical result, with one incarnation being found as [22, Proposition 11]. Our generalization is found as Theorem 11, showing that antagonistic games with finitely many outcomes have subgame-perfect equilibria.

3.2. A key example

As soon as we go beyond continuous payoff functions (or more generally, open preferences), Nash equilibria in infinite sequential games may fail to exist. We provide a generic folklore counterexample below, and will demonstrate that the underlying feature is essential for the failure of existence of Nash equilibria. The counterexample only requires a single player, and its payoff function is in a sense the least discontinuous payoff function, and in particular is $\Delta_{2}^{0}$-measurable.

Example 10. Let the payoff function $P:\{0,1\}^{\mathbb{N}} \rightarrow[0,1]$ for the single player be defined by $P\left(1^{n} 0 p\right)=\frac{n}{n+1}$ for all $p \in\{0,1\}^{\mathbb{N}}$ and $P\left(1^{\mathbb{N}}\right)=0$. As $P$ does not attain its supremum, the resulting game cannot have a Nash equilibrium.

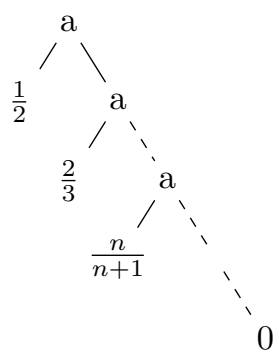


We will show in particular that the presence of a converging sequence of plays $\left(p^{n}\right)_{n \in \mathbb{N}}$ such that a player prefers $p^{n+1}$ to $p^{n}$, but prefers any $p^{n}$ to $\lim _{i \rightarrow \infty} p^{i}$, is a crucial feature of the example above to have no Nash equilibrium. The proof will be an adaption of the main result of [4 by the first author. Under the additional assumption of antagonistic preferences in a two-player game, we can even obtain subgame perfect equilibria.

\subsection{Results}

We state existence results for subgame perfect equilibria first, and then for Nash equilibria.

The restriction to antagonistic games here is motivated by [23, Example 3 ], which shows that even under very favourable conditions, non-antagonistic preferences lead to the non-existence of subgame-perfect equilibria. Our first result deals with the case of finitely many outcomes, such that for any history the set of its extensions yielding a particular outcome is determined. The most obvious way to ensure this condition is by having the set of plays yielding a particular outcome be (quasi)-Borel (Corollary 12).

Theorem 11. Let $\left\langle\{a, b\}, C, d, O, v,\left\{<,<^{-1}\right\}\right\rangle$ be an infinite sequential game where $O$ is finite and $<$ is a strict linear order. Let $\Gamma \subseteq \mathcal{P}\left(C^{\omega}\right)$ and assume the following.

1. $\forall O^{\prime} \subseteq O, \forall \gamma \in C^{*}, \quad\left\{\alpha \in C^{\omega} \mid v(\gamma \alpha) \in O^{\prime}\right\} \in \Gamma$

2. The game $\langle C, D, W\rangle$ is determined for all $W \in \Gamma$ and $D \subseteq C^{*}$.

Then the game $\left\langle\{a, b\}, C, d, O, v,\left\{<,<^{-1}\right\}\right\rangle$ has a subgame perfect equilibrium.

Corollary 12. Let $\left\langle\{a, b\}, C, d, O, v,\left\{<,<^{-1}\right\}\right\rangle$ be an infinite sequential game where $O$ is finite and $<$ is a strict linear order. If $v^{-1}(o)$ is quasi-Borel for all $o \in O$, the game has a subgame perfect equilibrium.

Proof. From Theorem 11, quasi-Borel determinacy 24], and Lemma 3.1. in [4. 
The statement of the second existence result for subgame perfect equilibrium involves the notion of guarantee of a player. This notion, also central in our proofs, was introduced in Definitions 2.3 and 2.5 from [4. The guarantee of a player is the smallest terminal interval, w.r.t her strict-weak-order preference, that includes the outcomes compatible with a given strategy of the player in the subgame at a given node of a given infinite sequential game. The best guarantee of a player consists of the intersection of all her guarantees over the set of strategies.

Definition 13 (Agent (best) guarantee). Let $\left\langle A, C, d, O, v,\left(\prec_{a}\right)_{a \in A}\right\rangle$ be a game where the $\prec_{a}$ are strict weak orders.

$$
\begin{aligned}
& \forall a \in A, \forall \gamma \in C^{*}, \forall s: d^{-1}(a) \rightarrow C, \quad g_{a}(\gamma, s):= \\
& \left\{o \in O \mid \exists p \in P\left(\left.s\right|_{\gamma C^{\omega}}\right) \cap \gamma C^{\omega}, v(p) \precsim_{a} o\right\} \\
& G_{a}(\gamma):=\bigcap_{s} g_{a}(\gamma, s)
\end{aligned}
$$

We write $g_{a}(s)$ and $G_{a}$ instead of $g_{a}(\gamma, s)$ and $G_{a}(\gamma)$ when $\gamma$ is the empty word.

We are now able to state the second existence result for subgame perfect equilibrium. Apart from the use of guarantees in the statement, a core difference is that Theorem 14 requires a finite set of actions, whereas Theorem 11 instead needs a finite set of outcomes. In particular, the strength of the two theorems is incomparable. Conditions 34 in Theorem 14 again constitute a determinacy requirement for the sets of plays yielding particular outcomes. Condition 2 is a technical condition stating that if a player can keep improving their guarantee along a path by changing strategies, then the outcome obtained on this path is included in all these guarantees. It can be seen as a very weak continuity assumption, which suffices to make out iterative construction of strategies wellbehaved.

Theorem 14. Let $\left\langle\{a, b\}, C, O, d, v,\left\{\prec, \prec^{-1}\right\}\right\rangle$ be a two-player antagonistic game, where $C$ is finite. Let $\Gamma \subseteq \mathcal{P}\left(C^{\omega}\right)$ and assume the following.

1. $\prec$ is a strict weak order. 
2. For every $p \in C^{\omega}$, sequence $\left(s_{n}\right)_{n \in \mathbb{N}}$ of strategies for $X \in\{a, b\}$, and increasing $\varphi: \mathbb{N} \rightarrow \mathbb{N}$, if $d\left(p_{<\varphi(n)}\right)=X$ and $g_{X}\left(p_{<\varphi(n+1)}, s_{n+1}\right) \subsetneq$ $g_{X}\left(p_{<\varphi(n)}, s_{n}\right)$ for all $n \in \mathbb{N}$, then $v(p) \in \cap_{n \in \mathbb{N}} g_{X}\left(p_{<\varphi(n)}, s_{n}\right)$.

3. For every $\precsim$-extremal interval $I$ and $\gamma \in C^{*}$, we have $\left(v^{-1}[I] \cap \gamma C^{\omega}\right) \in \Gamma$.

4. The game $\langle C, D, W\rangle$ is determined for all $W \in \Gamma, D \subseteq C^{*}$.

Then the game $\left\langle\{a, b\}, C, O, d, v,\left\{\prec, \prec^{-1}\right\}\right\rangle$ has a subgame perfect equilibrium. Moreover, for every $p$ as in Condition 2, one of the players controls only finitely many nodes on $p$, and after that her opponent plays as prescribed by $p$ in every SPE.

We now proceed to state existence results for Nash equilibria in multi-player games. Theorem 15 gives a very general (and technical) sufficient condition. Theorem 16 weakens and simplifies this sufficient condition to establish a characterization of $\mathrm{NE}$ existence in the original game as well as in some derived games. In Theorem 15. Conditions 223 again constrain the distribution of outcomes to determined sets. Condition 4 corresponds to Condition 2 in Theorem 14. Condition 5 states that each player has a strategy to realize their guarantee - these strategies are then used as the starting point for constructing a Nash equilibrium, by adding punishment for any deviators to it. Theorem 16 includes a way to construct such strategies if they are not already given.

Theorem 15. Let $\left\langle A, C, O, d, v,\left(\prec_{a}\right)_{a \in A}\right\rangle$ be a game, let $\Gamma \subseteq \mathcal{P}\left(C^{\omega}\right)$, and assume the following.

1. The $\prec_{a}$ are strict weak orders.

2. The game $\langle C, D, W\rangle$ is determined for all $W \in \Gamma, D \subseteq C^{*}$.

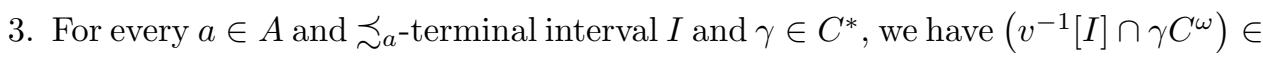
$\Gamma$.

4. For every play $p \in C^{\omega}$ and increasing sequence $\varphi: \mathbb{N} \rightarrow \mathbb{N}$, if $d\left(p_{<\varphi(n)}\right)=a$ and $G_{a}\left(p_{<\varphi(n+1)}\right) \subsetneq G_{a}\left(p_{<\varphi(n)}\right)$ for all $n \in \mathbb{N}$, then $v(p) \in \cap_{n \in \mathbb{N}} G_{a}\left(p_{<\varphi(n)}\right)$.

5. For all $\gamma \in C^{\omega}$, there exists $s$ such that $g_{a}(\gamma, s)=G_{a}(\gamma)$.

Then the game $\left\langle A, C, O, d, v,\left(\prec_{a}\right)_{a \in A}\right\rangle$ has a Nash equilibrium. 
Theorem 16 below is a simpler version of Theorem 15 that does only involve primitive notions from the definition of a game. Especially, it does not refer to the notion of guarantee. Via a necessary and sufficient condition, it shows how essential the feature of Example 10 is for the existence of Nash equilibrium. By pruning, we refer to choosing a subtree of the original game tree, and then working with the game restricted to this subtree. In other words, for each history we may prohibit certain actions to the agents.

Theorem 16. Let $g$ be a $\left\langle A, C, O, d, v,\left(\prec_{a}\right)_{a \in A}\right\rangle$ be a game where the $\prec_{a}$ are strict weak orders and $v$ is Borel-measurable. The following are equivalent.

1. For every $X \in A$ and $\left(p^{n}\right)_{n \in \mathbb{N}}$ sequence of plays in $C^{\omega}$ converging to some $p$, and increasing $\varphi: \mathbb{N} \rightarrow \mathbb{N}$, if for all $n \in \mathbb{N}$ we have $d\left(p_{<\varphi(n)}\right)=X$, $p_{<\varphi(n)}=p_{<\varphi(n)}^{n}, p_{\varphi(n)} \neq p_{\varphi(n)}^{n}$, and $v\left(p^{n}\right) \prec_{X} v\left(p^{n+1}\right)$, then $v\left(p^{n}\right) \prec_{X}$ $v(p)$ for all $n \in \mathbb{N}$.

2. Every finite-branching game derived from the original game by pruning has an NE.

340

However, the modification of Example 10 below shows that the conditions of Theorem 16 are not necessary for the mere existence of Nash equilibria in one specific game.

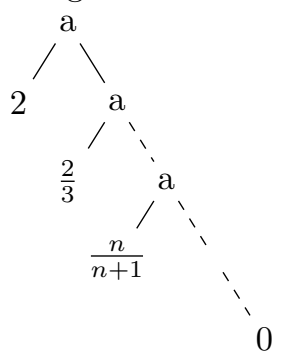

\subsection{Proofs}

Regarding the notion of guarantee, Lemma 2.4. from [4 still holds without major changes in the proofs, so we do not display it, but we collect some more useful facts in Observation 17 below. 
Observation 17. Let $\left\langle A, C, O, d, v,\left(\prec_{a}\right)_{a \in A}\right\rangle$, let $a \in A$, assume that $\prec_{a}$ is a strict weak order, and let $\gamma \in C^{*}$.

1. $d(\gamma) \neq a \Rightarrow G_{a}(\gamma)=\cup_{c \in C} G_{a}(\gamma \cdot c)$

2. $d(\gamma)=a \Rightarrow G_{a}(\gamma)=\cap_{c \in C} G_{a}(\gamma \cdot c)$

3. $d(\gamma)=a \wedge|C|<\infty \Rightarrow \exists c \in C, G_{a}(\gamma)=G_{a}(\gamma \cdot c)$

Proof. For example, for 2. note that $G_{a}(\gamma)=\cap_{s} g_{a}(\gamma, s)=\cap_{c \in C} \cap_{s(\gamma)=c} g_{a}(\gamma, s)=$ $\cap_{c \in C} \cap_{s} g_{a}(\gamma \cdot c, s)$

$355=\cap_{c \in C} G_{a}(\gamma \cdot c)$.

This section's proofs of existence of equilibria rely on each player having a (minimax-style) optimal strategy if all other players team up against her. Lemma 18 below provides a sufficient condition for such strategies to exist, i.e. for the best guarantee to be witnessed.

Lemma 18. Let $\left\langle A, C, O, d, v,\left(\prec_{a}\right)_{a \in A}\right\rangle$ be a game where $C$ is finite, let $a \in A$, and let us assume the following.

1. $\prec_{a}$ is a strict weak order.

2. For every play $p \in C^{\omega}$, increasing $\varphi: \mathbb{N} \rightarrow \mathbb{N}$, and sequence $\left(s_{n}\right)_{n \in \mathbb{N}}$ of strategies for Player $a$, if $g_{a}\left(p_{<\varphi(n+1)}, s_{n+1}\right) \subsetneq g_{a}\left(p_{<\varphi(n)}, s_{n}\right)$ for all $n \in \mathbb{N}$, then $v(p) \in \cap_{n \in \mathbb{N}} g_{a}\left(p_{<\varphi(n)}, s_{n}\right)$.

Then for all $\gamma \in C^{*}$ there exists $s \in S_{a}$ such that $g_{a}(\gamma, s)=G_{a}(\gamma)$.

Proof. W.l.o.g. we only prove that there exists $s \in S_{a}$ such that $g_{a}(s)=G_{a}$, i.e. where the $\gamma$ from the claim is the empty word. Let $s_{0}: d^{-1}(a) \rightarrow C$ be a strategy for Player $a$ and let us build inductively a sequence $\left(s_{n}\right)_{n \in \mathbb{N}}$ of strategies for Player $a$, as follows, where Case 3. implicitly invokes Observation 17.

- Let $\left.s_{n+1}\right|_{C<n}:=\left.s_{n}\right|_{C^{<n}}$.

- For all $\gamma \in C^{n} \backslash d^{-1}(a)$, let $\left.s_{n+1}\right|_{\gamma C^{*}}:=\left.s_{n}\right|_{\gamma C^{*}}$.

- For all $\gamma \in C^{n} \cap d^{-1}(a)$,

1. if $g_{a}\left(\gamma, s_{n}\right) \subseteq G_{a}$ then let $\left.s_{n+1}\right|_{\gamma C^{*}}:=\left.s_{n}\right|_{\gamma C^{*}}$, 
2. if $G_{a} \subsetneq g_{a}\left(\gamma, s_{n}\right)$ and there exists $\mu: d^{-1}(a) \cap \gamma C^{*} \rightarrow C$ such that $g_{a}(\gamma, \mu) \subseteq G_{a}$, let $\left.s_{n+1}\right|_{\gamma C^{*}}:=\mu$,

3. otherwise 4 let $s_{n+1}(\gamma):=c$ such that $G_{a}(\gamma \cdot c)=G_{a}(\gamma)$, and let $\left.s_{n+1}\right|_{\gamma C C^{*}}:=\left.s_{n}\right|_{\gamma C C^{*}}$.

Let $s$ be the limit strategy of the sequence $\left(s_{n}\right)_{n \in \mathbb{N}}$ and first note that, using Observation 17, one can prove by induction on $\gamma$ that $G_{a}(\gamma) \subseteq G_{a}$ for every $\gamma \in C^{*}$ that is compatible with $s$. Next, let $p \in P(s)$ be a path compatible with $s$. If $p$ has a prefix $\gamma$ that fell into Cases 1 . or 2 . during the recursive construction above, then $v(p) \in G_{a}$, so let us now assume that Case 3. applies at every node $p_{<n} \in d^{-1}(a)$. If such nodes are finitely many, let $p_{<n}$ be the deepest one, so ${ }_{385} d\left(p_{n+1+k}\right) \neq a$ for all $k \in \mathbb{N}$, and $v(p) \in g_{a}\left(p_{n+1}, t\right)$ for all strategies $t$ for $a$, so $v(p) \in G_{a}\left(p_{<n+1}\right)=\bigcap_{t} g_{a}\left(p_{<n+1}, t\right)$. So $v(p) \in G_{a}$ since $G_{a}=G_{a}\left(p_{<n+1}\right)$ by Case 3. Let us now assume that such nodes are infinitely many. If $G_{a}\left(p_{<n}\right) \subsetneq$ $G_{a}$ for some $p_{<n} \in d^{-1}(a)$, there exists $\mu: d^{-1}(a) \cap p_{<n} C^{*} \rightarrow C$ such that $G_{a}\left(p_{<n}\right) \subseteq g_{a}\left(p_{<n}, \mu\right) \subsetneq G_{a}$ since $G_{a}\left(p_{<n}\right)=\bigcap_{t} g_{a}\left(p_{<n}, t\right)$ by definition, which would mean that Case 1. or 2. applies; so $G_{a}\left(p_{<n}\right)=G_{a}$ for all $p_{<n} \in d^{-1}(a)$, and subsequently for all $n$. Also, the best guarantee is never witnessed (through Case 2.) at any node $p_{<n} \in d^{-1}(a)$, and subsequently for all $n$. If $v(p) \notin G_{a}$, the previous two remarks allow us to build inductively a sequence $\left(t_{n}\right)_{n \in \mathbb{N}}$ of strategies for $a$ such that $v(p) \notin g_{a}\left(p_{<0}, t_{0}\right)$ and $g_{a}\left(p_{<n+1}, t_{n+1}\right) \subsetneq g_{a}\left(p_{<n}, t_{n}\right)$ for all $n \in \mathbb{N}$, which would imply $v(p) \in \cap_{n \in \mathbb{N}} g_{a}\left(p_{<n}, t_{n}\right)$ by assumption of the lemma, contradiction.

Whereas Lemma 18 provides us with an optimal strategy for each history, we ultimately want a single strategy that is optimal everywhere. This is provided by 19 , which as Condition 3 starts with the conclusion in Lemma 18 . Note that Condition 2 in Lemma 19 is weaker than that in Lemma 18, and that finiteness of $C$ is used in Lemma 18 only. It is the main reason why Lemmas 18 and 19

\footnotetext{
${ }^{4}$ Note that due to the properties of a strict weak order, the sets of the form $g_{a}(\gamma, s)$ and $G_{a}(\gamma)$ are linearly ordered by inclusion $\subseteq$. Thus, $G_{a} \subsetneq g_{a}\left(\gamma, s_{n}\right)$ holds in this case, too.
} 
are not merged.

Lemma 19. Let $\left\langle A, C, O, d, v,\left(\prec_{a}\right)_{a \in A}\right\rangle$ be a game, let $a \in A$, and let us assume the following.

1. $\prec_{a}$ is a strict weak order.

2. For every play $p \in C^{\omega}$ and increasing $\varphi: \mathbb{N} \rightarrow \mathbb{N}$, if $d\left(p_{<\varphi(n)}\right)=a$ and $G_{a}\left(p_{<\varphi(n+1)}\right) \subsetneq G_{a}\left(p_{<\varphi(n)}\right)$ for all $n \in \mathbb{N}$, then $v(p) \in \cap_{n \in \mathbb{N}} G_{a}\left(p_{<\varphi(n)}\right)$.

3. For all $\gamma \in C^{*}$ there exists $s \in S_{a}$ such that $g_{a}(\gamma, s)=G_{a}(\gamma)$.

Then there exists $s$ such that $g_{a}(\gamma, s)=G_{a}(\gamma)$ for all $\gamma \in C^{*}$.

Proof. We proceed similarly as in the proof of Lemma 18. Let $s_{0}$ be a strategy for Player $a$ and let us build inductively a sequence $\left(s_{n}\right)_{n \in \mathbb{N}}$ of strategies for Player $a$. The recursive definition below is different from the one in the proof of Lemma 18 in three respects: the three occurrences of $G_{a}$ in Cases 1. and 2. are replaced with $G_{a}(\gamma)$. Case 3 . is deleted since it never applies by assumption. Finally, two inclusions are replaced with equalities.

- Let $\left.s_{n+1}\right|_{C<n}:=\left.s_{n}\right|_{C<n}$

- For all $\gamma \in C^{n} \backslash d^{-1}(a)$, let $\left.s_{n+1}\right|_{\gamma C^{*}}:=\left.s_{n}\right|_{\gamma C^{*}}$.

- For all $\gamma \in C^{n} \cap d^{-1}(a)$,

1. if $g_{a}\left(\gamma, s_{n}\right)=G_{a}(\gamma)$ then let $\left.s_{n+1}\right|_{\gamma C^{*}}:=\left.s_{n}\right|_{\gamma C^{*}}$,

420

2. if $G_{a}(\gamma) \subsetneq g_{a}\left(\gamma, s_{n}\right)$, let $\left.s_{n+1}\right|_{\gamma C^{*}}:=\mu$ where $\mu: d^{-1}(a) \cap \gamma C^{*} \rightarrow C$ is such that $g_{a}(\gamma, \mu)=G_{a}(\gamma)$.

Let $s$ be the limit strategy of the sequence $\left(s_{n}\right)_{n \in \mathbb{N}}$ and first note that, using Observation 17 , one can prove by induction on $\gamma$ that $G_{a}(\gamma) \subseteq G_{a}$ for every $\gamma \in C^{*}$ that is compatible with $s$. Next, let $p \in P(s)$ be a path compatible ${ }_{425}$ with $s$. Due to the uniformity of the recursive definition, it suffices to show that $v(p) \in G_{a}$ to prove the full statement.

If Case 2. applies only finitely many times in the construction of $s$, the sequence $\left(\left.s_{n}\right|_{\left\{\gamma \in C^{*} \mid p \in \gamma C^{\omega}\right\}}\right)_{n \in \mathbb{N}}$ is eventually constant, so $v(p) \in g_{a}\left(p_{<n}, s_{n}\right)=$ 
$G_{a}\left(p_{<n}\right) \subseteq G_{a}$ for some $n$. Otherwise, there exists an increasing function $\varphi$ : $\mathbb{N} \rightarrow \mathbb{N}$ with $d\left(p_{<\varphi(n)}\right)=a$ and $G_{a}\left(p_{<\varphi(n+1)}\right) \subsetneq G_{a}\left(p_{<\varphi(n)}\right)$ for all $n \in \mathbb{N}$, so $v(p) \in \cap_{n \in \mathbb{N}} G_{a}\left(p_{<\varphi(n)}\right) \subseteq G_{a}\left(p_{<\varphi(0)}\right) \subseteq G_{a}$.

Lemma 20. Let $\left\langle\{a, b\}, C, O, d, v,\left\{\prec, \prec^{-1}\right\}\right\rangle$ be a two-player game. Let $\Gamma \subseteq$ $\mathcal{P}\left(C^{\omega}\right)$ and assume the following.

1. $\prec$ is a strict weak order.

2. For every play $p \in C^{\omega}$ and increasing sequence $\varphi: \mathbb{N} \rightarrow \mathbb{N}$, if $d\left(p_{<\varphi(n)}\right)=a$ and $G_{a}\left(p_{<\varphi(n+1)}\right) \subsetneq G_{a}\left(p_{<\varphi(n)}\right)$ for all $n \in \mathbb{N}$, then $v(p) \in \cap_{n \in \mathbb{N}} G_{a}\left(p_{<\varphi(n)}\right)$.

3. For all $\gamma \in C^{\omega}$, there exists $s$ such that $g_{a}(\gamma, s)=G_{a}(\gamma)\left(\operatorname{resp} . g_{b}(\gamma, s)=\right.$ $\left.G_{b}(\gamma)\right)$.

4. For all non-empty closed $E \subseteq C^{\omega}$, there are $\precsim$-extremal elements in $v[E]$.

5. For every $\precsim$-extremal interval $I$ and $\gamma \in C^{*}$, we have $\left(v^{-1}[I] \cap \gamma C^{\omega}\right) \in \Gamma$.

6. The game $\langle C, D, W\rangle$ is determined for all $W \in \Gamma, D \subseteq C^{*}$.

Then the game $\left\langle\{a, b\}, C, O, d, v,\left\{\prec, \prec^{-1}\right\}\right\rangle$ has a subgame perfect equilibrium.

Proof. By invoking Lemma 19 once for Player $a$ and once for Player $b$, let us build a strategy profile $s: C^{*} \rightarrow C$, such that $g_{X}\left(\gamma, s_{X}\right)=G_{X}(\gamma)$ for all ${ }_{445} \gamma \in C^{*}$ and $X \in\{a, b\}$. Let $\gamma \in C^{*}$ and let us prove that $G_{a}(\gamma) \cap G_{b}(\gamma)=$ $\left\{\min _{<}\left(G_{a}(\gamma)\right)\right\}=\left\{\max _{<}\left(G_{b}(\gamma)\right)\right\}$. Consider the game $\langle C, D, W\rangle$ (as in Definition 5 5 where the winning set is defined by $W:=\left\{\alpha \in \gamma C^{\omega} \mid v(\alpha) \in\right.$ $\left.G_{a}(\gamma) \backslash\left\{\min _{<}\left(G_{a}(\gamma)\right)\right\}\right\}$ and where Player $a$ owns exactly the nodes in $D:=$ $\left(C^{*} \backslash \gamma C^{*}\right) \cup\left(d^{-1}(\{a\}) \cap \gamma C^{*}\right)$. By Assumption 5 the set $W$ is in $\Gamma$, so by Assumption 6 the game $\langle C, D, W\rangle$ is determined. By definition of the best guarantee, Player $a$ has no winning strategy for this game, so Player $b$ has a winning strategy, which means that $G_{b}(\gamma) \subseteq\left\{\min _{<}\left(G_{a}(\gamma)\right)\right\} \cup O \backslash G_{a}(\gamma)$. Since $G_{a}(\gamma) \cap G_{b}(\gamma)$ must be non-empty, otherwise the two guarantees are contradictory, $G_{a}(\gamma) \cap G_{b}(\gamma)=\left\{\min _{<}\left(G_{a}(\gamma)\right)\right\}$. This means that the subprofile of $s$ rooted at $\gamma$ induces the outcome $\min _{<}\left(G_{a}(\gamma)\right.$ ) (which equals $\max _{<}\left(G_{b}(\gamma)\right.$ ) by symmetry), and it is optimal for both players.

We now have the ingredients to prove our theorems: 
Proof of Theorem 14. By application of Lemma 20. (Note that Condition 2 of Theorem 14 implies both Condition 2 of Lemma 18 and Condition 2 of Lemma 19.) Condition 3 is proved by Lemma 18 For Condition 4 , let $E$ be a non-empty closed subset of $C^{\omega}$, and let $T$ be the tree such that $[T]=E$. Consider the game where Player $a$ plays alone on $T$. Since Player $a$ can maximise her best guarantee by Lemma 18 , and since all her guarantees are singletons, $v[E]$ has a $\prec$-maximum. Likewise, it has a $\prec$-minimum, by considering Player ${ }_{465} b$.

Proof of Theorem 11 . by Lemma 20 where Conditions 2,3 , and 4 hold by finiteness of $O$.

Proof of Theorem 15. Since the proof is similar to that of Theorem 2.9 in [4, we rephrase and give it a more intuitive flavour. Let $\sigma$ be a strategy profile where every player is using a witness to Lemma 19 . Let $p$ be the induced play. We now turn $\sigma$ into a Nash equilibrium with $p$ as induced play by use of threats. More specifically, at each node $p_{<n}$ we let the players other than $a:=d\left(p_{<n}\right)$ threaten Player $a$ that if she deviates from $p$ exactly at $p_{<n}$, they will team up against her at every subsequent position $\gamma$ after $p_{<n}$ other than those extending 475 the prescribed $p_{<n+1}$.

We claim that if they team up, they can prevent Player $a$ from getting better outcome than $v(p)$ by deviating to $\gamma$, which will suffice. Let us build a win/lose game $\langle C, D, W\rangle$, with Player $a$ against her threatening opponents gathered as a meta-player, and where the winning set for Player $a$ is defined ${ }_{480}$ by $W=v^{-1}[I] \cap \gamma C^{\omega}$, where $I:=\left\{o \in O \mid v(p) \prec_{a} o\right\}$, and $D$ is defined by $D=d^{-1}(\{a\}) \cup\left(C^{*} \backslash \gamma C^{*}\right)$. This game is determined by Assumptions 2 and 3 , and Player $a$ loses it, otherwise her winning strategy would guarantee that $v(p) \notin G_{a}\left(p_{<n}\right)$ and thus contradict the choice of $p$. Therefore the threat of the opponents of Player $a$ is effective.

Proof of Theorem 16. Let us first prove 1. $\Rightarrow 2$. by invoking Theorem 15 . More specifically, let $T$ be a finite-branching, infinite subtree of $C^{\omega}$ and consider the 
restriction of the original game to $T$. Conditions 3 and 2 follow from Borel measurability and [1]. Condition 5 comes from Lemma 18 (actually a straightforward extension of Lemma 18 to trees with finite-yet-unbounded branching), and Condition 4 follows directly from the assumption.

For 2. $\Rightarrow 1$., let $X \in\{a, b\}$ and let $\left(p^{n}\right)_{n \in \mathbb{N}} \rightarrow p \in C^{\omega}$ and increasing $\varphi: \mathbb{N} \rightarrow \mathbb{N}$ such that for all $n \in \mathbb{N}$ we have $d\left(p_{<\varphi(n)}\right)=X, p_{<\varphi(n)}=p_{<\varphi(n)}^{n}$, $p_{\varphi(n)} \neq p_{\varphi(n)}^{n}$, and $v\left(p^{n}\right) \prec_{X} v\left(p^{n+1}\right)$. Let $T$ be the tree made of the prefixes of $p$ and the $p^{n}$. Since the game induced by $T$ has an NE and its tree structure is similar to Example $10, v\left(p^{n}\right) \prec_{X} v(p)$ must hold for all $n \in \mathbb{N}$.

\subsection{Further discussion}

For further comparison, the preparatory work before [4, Theorem 2.9] considers strict well-orders only; then [4, Theorem 2.9] considers strict well-founded orders, since linear extensions of these make it possible to invoke the special, linear case, knowing that any Nash equilibrium for these extensions is still a Nash equilibrium for the original preferences. However, let us explain why Theorem 15 assumes that preferences are strict weak orders, instead of more general strict partial orders. In the preparatory work before both results, the algorithm that builds a play step by step makes decisions based on the guarantees that the subgames offer. If the guarantees of one player were not ordered by a strict weak order, the player might eventually regret a previous decision, in the same way that backward induction on partially ordered preferences may not yield a Nash equilibrium (see e.g., 25] for a concrete example or page 3 of [26] for a generic one). So the algorithm has to run on strict weak orders. (In 4, Theorem $2.9]$ it even runs on strict linear orders.)

If we wanted to consider strict partial orders and extend them linearly for the algorithm to work, we would potentially run into two problems: first, there may not exist any linear extension preserving Condition 4. Second, assumptions 2 and 3 of Theorem 15 make sure that the win/lose games associated with the $\precsim a^{-}$ terminal intervals are determined, which is a requirement for the proof to work. If the preferences were not strict weak orders, we might think of replacing the 
condition on terminal intervals by a condition on the upper-closed sets and then extend the preferences linearly for the algorithm to work, but in the special case where the preference of one player were the empty relation, every subset would be an upper-closed set and its preimage by $v$ would be in the pointclass with nice closure property, by assumption. If, in addition, each outcome is mapped to at most one play, it implies that each subset of $C^{\omega}$ is in the pointclass, so Theorem 15 could be used with the axiom of determinacy only, but not with, e.g., Borel determinacy. On the contrary, 4, Theorem 2.9, Assumption 3] is not an issue since there are only countably many outcomes in that setting.

The results in this section are generally not constructive - but neither is Nash's theorem in [27, cf. [28, 29]. The extent of non-constructivity is investigated in 30.

The condition on the payoff functions used in Theorems 15, 16 seems to merit further investigation. This was that for any sequence $\left(p^{i}\right)_{i \in \mathbb{N}}$ converging to $p$ in $C^{\omega}$, we find that $\forall i \in \mathbb{N} v\left(p^{i}\right) \prec v\left(p^{i+1}\right)$ implies $\forall i \in \mathbb{N} v\left(p^{i}\right) \prec v(p)$. This is a weaker condition than continuity of the function where the upper order topology is used on the codomain, which still seems to be strong enough to formulate some results. In a sense, it is a weakening of continuity that is orthogonal to Borel-measurability. As an example, a result by GREGORIADES (reported in [31]) shows that any function of this type from Baire space to the countable ordinals has to be bounded.

\section{On the existence of Pareto-optimal NE and secure equilibria}

\subsection{On the existence of Pareto-optimal NE}

In this section we investigate very general classes of games that guarantee existence of Nash equilibria, and such that there exists an NE that is Paretooptimal among all the profiles of the game (not just Pareto-optimal among all Nash equilibria). In the following, we shall assume that any outcome is realizable to avoid unnecessary case-distinctions. 

$\langle C, D, W\rangle$ is determined for all $W \in \Gamma, D \subseteq C^{*}$. Given some preferences $\left(\prec_{a}\right)_{a \in A}$ on outcomes $O$, we say that a function $f: C^{\omega} \rightarrow O$ is $\Gamma$-measurable, if for any $a \in A$ and $o \in O$ we find that $f^{-1}\left(\left\{o^{\prime} \in O \mid o^{\prime} \prec o\right\}\right) \in \Gamma$.

Theorem 21. We fix a non-empty set of players $A$ and a non-empty set of can enforce some upper interval. As long as the opponent can enforce $y$, he can force the controller to play along the chosen path by threaten punishment by $y$ for deviation. If we ever reach a vertex where the controller (w.l.o.g. $a$ ) can 
enforce $\left\{x_{1}, \ldots, x_{n}\right\}$, there will be some minimal upper set $\left\{x_{i}, \ldots, x_{n}\right\}$ (from enforce $\left\{y, x_{1}, \ldots, x_{i}\right\}$. We then let both players play their enforcing strategy from this node onwards.

The constructed partial strategies can be extended in an arbitrary way to yield a Nash equilibrium with another outcome than $y$, and these are all Pareto-

Proof of Theorem 21.

3. $\Rightarrow$ 2. Clear.

2. $\Rightarrow 1$. By contraposition, let us assume that $z \prec_{a} y \prec_{a} x$ and $x \prec_{b} z \prec_{b} y$, and note that the game below has only one NE yielding outcome $z$.

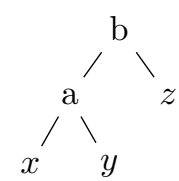

1. $\Rightarrow 3$. By $[32$, Lemma 4] the second assumption in 1 . implies that there exists a partition $\left\{O_{i}\right\}_{i \in I}$ of $O$ and a linear order $<$ over $I$ such that $i<j$ implies $x<_{a} y$ for all $a \in A$ and $x \in O_{i}$ and $y \in O_{j}$, and such that $<_{b}\left|O_{i}=<_{a}\right|_{O_{i}}$ or $<\left._{b}\right|_{O_{i}}=<\left._{a}\right|_{O_{i}} ^{-1}$ for all $a, b \in A$. By the well-foundedness assumption, $I$ has a $<-$ maximum $m$.

Fix some $a \in A$, let $\left\{x_{1}, \ldots, x_{n}\right\}:=O_{m}$ (again, by well-foundedness, each slice is finite) such that $x_{n}<_{a} \cdots<_{a} x_{1}$, let $A_{0}:=\left\{b \in A\left|<_{b}\right|_{O_{m}}=<_{a}\right.$ $\left.\left.\right|_{O_{m}}\right\}$, let $A_{1}:=A \backslash A_{0}$, and let $y \notin O_{m}$. Let us derive a new game on the same tree: each vertex of the original game owned by $b \in A$ is now owned by $A_{0}$ if $b \in A_{0}$ and by $A_{1}$ otherwise. Each play of the original game that induces an outcome outside of $O_{m}$ induces $y$ in the derived game. The new preferences are $y<A_{0} x_{n}<_{A_{0}} \cdots<_{A_{0}} x_{1}$ and $y<{A_{1}}_{1} x_{1}<_{A_{1}} \cdots<_{A_{1}} x_{n}$. By Lemma 22, the derived game has a Pareto-optimal NE (which cannot yield $y$, as this is the only non-Pareto-optimal outcome). It is also a Pareto-optimal NE for the original game. 
The situation for non-linear orders is less clear. Certainly, whenever some linearization avoids the forbidden pattern from Theorem 21 (1.), there will be a Pareto-optimal NE (as being Pareto-optimal w.r.t. the linearization implies being Pareto-optimal w.r.t. the original preferences). However, we do not know whether partial preferences such that any linearization has the forbidden pattern is enough to enable absence of Pareto-optimal NE. Two examples that could potentially play a similar role to the generic counterexample in Theorem 21 $(2 . \rightarrow 1$.) follow:

Example 23. We consider a finite two-player game with outcomes $\{x, y, z, \alpha, \beta, \gamma\}$, preferences $\gamma \prec_{a} y \prec_{a} x$ and $z \prec_{a} \beta \prec_{a} \alpha$ and $x \prec_{b} z \prec_{b} y$ and $\alpha \prec_{b} \gamma \prec_{b} \beta$ and game tree:

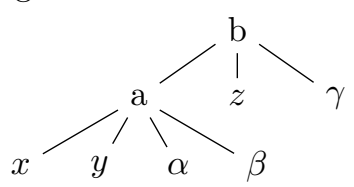

The preferences avoid the forbidden pattern from Theorem 21 (1.); but the pattern is present in any linear extension. In the Nash equilibria of the game, player $b$ is choosing either $z$ or $\gamma$; and player $a$ is choosing $x$ or $\alpha$. In particular, the potential equilibrium outcomes are $z$ and $\gamma$-precisely those outcomes that are not Pareto-optimal (because every player prefers $y$ to $z$ and $\beta$ to $\gamma$ ).

Example 24. We consider a finite two-player game with outcomes $\{x, y, z, t\}$, preferences $t, z \prec_{a} x, y$ and $x \prec_{b} z \prec_{b} y \prec_{b} t$ and game tree:

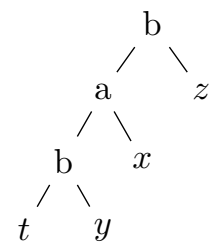

The preferences are strict weak orders and avoid the forbidden pattern from Theorem 21 (1.); but the pattern is present in any linear extension. The only 
equilibrium outcome is $z$, despite everyone preferring $y(5)$.

A related notion to Pareto-optimal Nash equilibria are the strong Nash equilibria introduced by Aumann [35]. These are the strategy profile where no coalition of players can deviate in a manner than benefits all of them. A characterization of the games admitting strong Nash equilibria has been elusive so far, though some recent progress was made by Nessah and Tian [36] in the setting of games in normal form.

In fact, Theorem 21 remains true if Pareto-optimal Nash equilibrium is replaced by strong Nash equilibrium 6 The counterexample showing that the condition on the preferences is necessary also fails to be strong Nash equilibrium, since the coalition of both players can profitably deviate from $z$ to $y$. Conversely, if the condition on the preferences are satisfied, then the outcomes can be sorted into layers in a way that all players agree on the preferences between layers, and all players either have one particular linear order of the outcomes inside each layer or its opposite. We can safely merge all players that agree on their preferences inside the best possible layer into a single player. A Paretooptimal Nash equilibrium in the resulting game is a strong Nash equilibrium in the original game.

\subsection{On the existence of secure equilibria}

This subsection shows that the construction of the malevolent preferences preserves several order-theoretic properties of the preferences. These preservation results allow us to invoke existence theorems for NE for the malevolent preferences (Definition 9) and thus to prove existence of secure equilibria in several general settings. Most of the previous work on the existence of secure equilibria was focused on very specific classes of games (e.g. [16, 37, 14]), with

\footnotetext{
${ }^{5}$ It may be an interesting remark that in this game every player would benefit, if $b$ could not choose $t$ at his second move.

${ }^{6}$ We are grateful to a referee of a previous version for raising the question we are answering here.
} 
the exception of [38]. We show that the second main theorem of [38] can be obtained as a corollary with our methods, and provide some more general existence results (Corollaries 29, 31, 33.

Consider some preferences $\left(\prec_{a}\right)_{a \in A}$, and let $\left(\prec_{a}^{\prime}\right)_{a \in A}$ be the induced malevolent preferences used to define secure equilibria.

Lemma 25. If the $\left(\prec_{a}\right)_{a \in A}$ are strict weak orders, the $\left(\prec_{a}^{\prime}\right)_{a \in A}$ are irreflexive 655 and transitive.

Proof. Irreflexivity is easy to check since $\prec_{a}$ is a strict weak order for all $a \in A$, so let us focus on transitivity. Let $a \in A$ and $x, y, z \in O$ be such that $x \prec_{a}^{\prime}$ $y \prec_{a}^{\prime} z$. If $x \prec_{a} z$ then $x \prec_{a}^{\prime} z$ and we are done, so let us assume that $z \precsim a x$. Clearly, $x \prec_{a}^{\prime} y \prec_{a}^{\prime} z$ implies $x \precsim_{a} y \precsim_{a} z$, so $x \sim_{a} y \sim_{a} z$. Since for all $b \in A$ we have $y \precsim_{b} x$ and $z \precsim_{b} y$, we find $z \precsim_{b} x$. Moreover there exists $c \in A$ such that $y \prec_{c} x$, so $z \prec_{c} x$. Therefore $x \prec_{a}^{\prime} z$.

However, the malevolent construction does not preserve strict weak orders, as is shown by the following example where the first components are for player $a:(0,2,0) \prec_{a}(0,1,0)$, whereas $(0,0,1)$ is comparable with neither $(0,1,0)$ nor $(0,2,0)$ as far as player $a$ is concerned. Fortunately, there are results of existence of NE that do not assume strict weak order preferences, namely:

Theorem 26 ([4, Theorem 3.2.]). Let $A$ be a non-empty set of players, $C$ a set of actions with at least 2 elements, let $O$ be a non-empty countable set of outcomes. For each $a \in A$, let $\prec_{a}$ be a binary relation on $O$. Then the following are equivalent:

1. All $\prec_{a}^{-1}$ are well-founded.

2. For any assignment of players $d: C^{*} \rightarrow A$ and any (quasi)-Borel measurable outcome function $v: C^{\omega} \rightarrow O$ the game $\left\langle A, C, d, O, v,\left(\prec_{a}\right)_{a \in A}\right\rangle$ has a Nash equilibrium.

We can immediately rediscover [38, Theorem 2]: 
Corollary 27. Let $g$ be a $\left\langle A, C, O, d, v,\left(\prec_{a}\right)_{a \in A}\right\rangle$ be a game where the $\prec_{a}$ are strict weak orders, $O$ is finite and $v$ is Borel-measurable. Then $g$ has a secure equilibrium.

Proof. Let us derive $g^{\prime}$ from $g$ by using the malevolent preferences $\prec_{a}^{\prime}$. They are strict partial orders by Lemma 25, so by Theorem $26 g^{\prime}$ has a Nash equilibrium.

What makes it difficult to generalize Corollary 27 to infinite-range outcome functions is that even if all the $\prec_{a}$ have well-founded inverses, it may no longer be the case for the malevolent $\prec_{a}^{\prime}$. Indeed, consider the payoff pairs $\left(0, \frac{1}{n+1}\right)$ for all $n \in \mathbb{N}$. We circumvent this issue in three different ways below. First we use a well-known special case of well-foundedness: a well-quasi order is a well-founded order with no infinite anti-chains. (Where an anti-chain is a set of elements that are pairwise non-comparable.)

Lemma 28. Let $\left(\prec_{a}\right)_{a \in A}$ be strict weak orders. If their inverses are well-quasi orders, so are the inverses of the $\prec_{a}^{\prime}$.

Proof. Let us first agree to write $x \sim_{a}^{\prime} y$ to denote $\neg\left(x \prec_{a}^{\prime} y\right) \wedge \neg\left(y \prec_{a}^{\prime} x\right)$ even though $\prec_{a}^{\prime}$ may not be a strict weak order. For all $x, y$, if $x \sim_{a}^{\prime} y$ then $x \sim_{a} y$, by definition of $\prec_{a}^{\prime}$. So the $\prec_{a}^{\prime}$ have no infinite anti-chains. Since every linear extension of a well-quasi order is a well-order, the $\prec_{a}^{\prime}$ have no infinite ascending chains.

Corollary 29 below does not exploit Lemma 28 fully: It only uses the wellfoundedness part of its conclusion.

Corollary 29. Let $\left(\prec_{a}\right)_{a \in A}$ be strict weak orders whose inverses are well-quasi orders. All games with the $\left(\prec_{a}\right)_{a \in A}$ and Borel-measurable outcome functions have secure equilibria.

Proof. By Lemma 28 and Theorem 26.

Lemma 30 below weakens the assumption by allowing infinite anti-chains. 
Lemma 30. Let $\left(\prec_{a}\right)_{a \in A}$ be strict weak orders such that no antichain of some $\prec_{a}$ is an infinite descending chain of $\cup_{b \in A} \prec_{b}$. If the $\prec_{a}$ have no infinite ascending chains, neither have the $\prec_{a}^{\prime}$.

Proof. Towards a contradiction let $x_{0} \prec_{a}^{\prime} x_{1} \prec_{a}^{\prime} x_{2} \ldots$ be an infinite chain. By well-foundedness of $\prec_{a}^{-1}$, there exists $k$ such that $x_{n} \sim_{a} x_{k}$ for all $n \geq k$. So for all $n \geq k$ we have $x_{n+1} \prec_{b_{n}} x_{n}$ for some $b_{n}$, contradiction.

Corollary 31 below invokes Lemma 30 Its assumptions refer to the preferences collectively (as one big relation), which may not sound as usual as a conjunction of individual conditions. However, note that the malevolent preferences already combine the original preferences, by definition.

Corollary 31. Let $\left(\prec_{a}\right)_{a \in A}$ be strict weak orders such that no antichain of some $\prec_{a}$ is an infinite descending chain of $\cup_{b \in A} \prec_{b}$. If the $\prec_{a}$ have no infinite ascending chains, games with the $\prec_{a}$ and Borel measurable outcome functions have secure equilibria.

Proof. By Lemma 30 and Theorem 26.

If one is willing to work with a finite number of players, one can further weaken the assumption on the descending chains (compared to Lemma 30), into a conjunction of individual conditions on the preferences. We thus obtain a better preservation result in Lemma 32 below.

Lemma 32. Let $A$ be finite and let $\left(\prec_{a}\right)_{a \in A}$ be strict weak orders. Let us assume that

1. no $\prec_{a}$ has infinite ascending chains, and

2. no antichain of some $\prec_{a}$ is an infinite descending chain of some $\prec_{b}$.

Then the $\prec_{a}^{\prime}$ satisfy the same two assumptions.

Proof. Let us prove the two properties in the same order.

1. Towards a contradiction let $x_{0} \prec_{a}^{\prime} x_{1} \prec_{a}^{\prime} x_{2} \ldots$ be an infinite chain. By well-foundedness of $\prec_{a}^{-1}$, there exists $k$ such that $x_{n} \sim_{a} x_{k}$ for all $n \geq k$. 
So for all $n \geq k$ we have $x_{n+1} \precsim_{b} x_{k}$ for all $b$ and $x_{n+1} \prec_{b_{n}} x_{n}$ for some $b_{n}$. By finiteness of $A$, there exists $b \in A$ and a subsequence $b_{\varphi(n)}$ such that $b_{\varphi(n)}=b$ for all $n$. So $x_{\varphi(n+1)} \prec_{b} x_{\varphi(n)}$ for all $n$, contradiction.

2. Towards a contradiction let $x_{0} \succ_{b}^{\prime} x_{1} \succ_{b}^{\prime} x_{2} \ldots$ be such that $x_{i} \sim_{a}^{\prime} x_{j}$ for all $i, j$. Therefore $x_{i} \sim_{a} x_{j}$ for all $i, j$, and by the second property only finitely many of the $x_{n+1} \prec_{b}^{\prime} x_{n}$ come from $x_{n+1} \prec_{b} x_{n}$. So, for all $n$ there exists $c_{n}$ such that $x_{n+1} \prec_{c_{n}} x_{n}$. By finiteness of $A$, this contradicts the second property.

It would not be possible to just drop the assumption that the players are finitely many from Lemma 32 , as is shown by the following example: Let $\left(u_{n}\right)_{n \in \mathbb{N}}$ be a family of infinite binary sequences such that the first $n+1$ members of $u_{n}$ are 0 's, and the rest are 1's. For instance, $u_{2}=000111111 \ldots$ Take each $u_{n}$ as a payoff tuple for infinitely many players. All chains the corresponding order have at most two elements, so there are definitely no infinite chains. However,

$745 u_{n} \prec_{1}^{\prime} u_{n+1}$ for all $n$, where $\prec_{1}$ is the preference of the first player, who always receives payoff 0 .

Similarly to Corollary 29, the preservation Lemma 32 makes it easy to prove another result of existence of secure equilibria.

Corollary 33. Let $A$ be finite and $\left(\prec_{a}\right)_{a \in A}$ be strict weak orders such that no $\prec_{a}$ has infinite ascending chains, and no anti-chain of some $\prec_{a}$ is an infinite descending chain of some $\prec_{b}$. Then every game with preferences $\left(\prec_{a}\right)_{a \in A}$ and with Borel-measurable outcome function has a secure equilibrium.

Proof. By Lemma 32 the malevolent preferences have no infinite ascending chains, so we conclude by Theorem 26 .

We would like to generalize further the existence of secure equilibria even in the presence of infinite ascending chains in the preferences, as in and by invoking Theorem 16. However, the malevolent construction does not preserve 
strict weak orders, as is shown in the beginning of the section, so we would need to extend our NE existence result beforehand.

\section{Corollaries on real-valued payoff functions}

We shall briefly discuss corollaries about games with real-valued payoff functions, which we obtain from our main theorems. In some cases, this requires to make statements about $\varepsilon$-Nash equilibria or $\varepsilon$-subgame perfect equilibria instead of Nash equilibria or subgame-perfect equilibria to satisfy the criteria of the theorems.

Theorem 15 has a corollary pertaining to sequential games with real-valued payoffs. Recall that a payoff-function $P:\{0,1\}^{\mathbb{N}} \rightarrow \mathbb{R}$ is called upper semicontinuous, if whenever $\left(p^{n}\right)_{n \in \mathbb{N}}$ is a converging sequence of plays, then $P\left(\lim _{i \in \mathbb{N}} p^{i}\right) \geq$ $\limsup _{i \in \mathbb{N}} P\left(p^{i}\right)$. In particular, Condition 4 in Theorem 15 is always satisfied for the preferences obtained from upper semi-continuous payoff functions.

Corollary 34. Sequential games with countably many players, finitely many choices and upper-semicontinuous payoff functions have Nash equilibria.

A rather simple argument allows us to transfer existence theorems for equilibria in games with Borel-measurable valuations to Borel-measurable real-valued payoff functions with upper bound, if one is willing to replace the original notions by their $\varepsilon$-counterparts. If $v: \mathbf{S} \rightarrow(-\infty, 0]^{\omega}$ is the Borel-measurable payoff function (with a component for each of the countably many players), then for every positive real $\epsilon$ we define $v_{\epsilon}: \mathbf{S} \rightarrow \mathbb{N}^{\omega}$ by $v_{\epsilon}^{-1}\left(\left(i_{k}\right)_{k \in \mathbb{N}}\right):=$ $\left.\left.\left.\left.v^{-1}(]-\left(i_{1}+1\right) \epsilon, i_{1} \epsilon\right] \times\right]-\left(i_{2}+1\right) \epsilon, i_{2} \epsilon\right] \times \ldots\right)$. Then any $v_{\epsilon}$ is again a Borel measurable valuation (as a product of countably many intervals is $\Pi_{2}^{0}$ ). Furthermore, we define the preferences $\prec_{n}$ for the $n$-th player by $\left(i_{k}\right)_{k \in \mathbb{N}} \prec_{n}\left(j_{k}\right)_{k \in \mathbb{N}}$ iff $i_{n}<j_{n}$. Now every Nash equilibrium of the resulting game is a $\epsilon$-Nash equilibrium of the original game, and every subgame perfect equilibrium of the resulting game is a $\epsilon$-subgame perfect equilibrium of the original game. 
Corollary 35. 7 Sequential games with countably many players and Borelmeasurable upper-bounded payoff functions admit $\varepsilon$-Nash equilibria.

Proof. By combining the statement of Theorem 16 with the argument above. We can invoke Theorem 16 as the preferences $\prec_{n}$ do not have any infinite ascending chains at all.

From Theorem 11 we obtain the following:

Corollary 36. An antagonistic game with a Borel-measurable bounded payoff function has an $\varepsilon$-subgame perfect equilibrium.

In a game with payoff functions $\left(p_{a}\right)_{a \in A}$, let us call an outcome $v \varepsilon$-Pareto optimal, if there is no realizable outcome $v^{\prime}$ and player $a_{0}$ such that $p_{a_{0}}\left(v^{\prime}\right) \geq$ $p_{a_{0}}(v)+\varepsilon$ and for any $a, p_{a}\left(v^{\prime}\right) \geq p_{a}(v)$. Then from Theorem 21 we can conclude:

Corollary 37. Sequential games with countably many players and Borel-measurable upper-bounded payoff functions $\left(p_{a}\right)_{a \in A}$ such that there are no two players $a$ and $b$ and three outcomes $x, y, z$ such that $p_{a}(x)>p_{a}(y)>p_{a}(z)$ and $p_{b}(y)>p_{b}(z)>p_{b}(x)$ admit $\varepsilon$-Pareto optimal $\varepsilon$-Nash equilibria.

Related work concerning games with finitely many players includes [18, Corollary 4.2] showing that games with continuous payoff functions have subgame perfect equilibria, [8, Theorem 2.1] showing that upper-semicontinuous payoff functions yield $\varepsilon$-subgame perfect equilibria 8 , 7, Theorem 2.3$]^{9}$ showing that also lower-semicontinuous payoff functions yield $\varepsilon$-subgame perfect equilibria.

\footnotetext{
${ }^{7}$ In his survey 39, MerTEns sketches an observation by himself and NEYMAN that one may use Borel determinacy to directly obtain the special case of this result for finitely many players and bounded payoffs.

${ }^{8}$ In [3] we had raised the question whether this result generalizes to countably many players. A positive answer was since given by Flesch and Predtetchinski 40.

${ }^{9}$ As shown in [7, Subsection 4.3], there are games with countably many players and lowersemicontinuous payoff functions without $\varepsilon$-subgame perfect equilibria.
} 
For a game with $\Delta_{2}^{0}$-measurable payoff functions avoiding the preference pattern from the Solan-Vieille counterexample [23], it is shown in [32, Corollary $2]$ that the game has a Pareto-optimal subgame perfect equilibrium.

\section{Acknowledgments.}

We are very grateful to Vassilios Gregoriades and the anonymous referees of this and the conference version for their helpful comments. This work benefited from the Royal Society International Exchange Grant IE111233. The authors participated in the Marie Curie International Research Staff Exchange Scheme Computable Analysis, PIRSES-GA-2011- 294962, and are also supported by the ERC inVEST (279499) project.

[1] D. A. Martin, Borel determinacy, Annals of Mathematics 102 (2) (1975) pp. 363-371. doi:10.2307/1971035.

[2] S. Le Roux, A. Pauly, Extending finite memory determinacy, Information and Computationdoi:10.1016/j.ic.2018.02.024.

[3] S. Le Roux, A. Pauly, Infinite sequential games with real-valued payoffs, in: CSL-LICS '14, ACM, 2014, pp. 62:1-62:10. doi:10.1145/2603088. 2603120

[4] S. Le Roux, Infinite sequential Nash equilibria, Logical Methods in Computer Science 9 (2). doi:10.2168/LMCS-9(2:3) 2013.

[5] M. Ummels, Rational behaviour and strategy construction in infinite multiplayer games, Diploma thesis, RWTH Aachen (2005).

[6] E. Grädel, M. Ummels, Solution concepts and algorithms for infinite multiplayer game, in: K. Apt, R. van Rooij (Eds.), New Perspectives on Games and Interaction, Vol. 4 of Texts in Logic and Games, Amsterdam University Press, 2008, pp. 151-178. 
[7] J. Flesch, J. Kuipers, A. Mashiah-Yaakovi, G. Schoenmakers, E. Solan, K. Vrieze, Perfect-information games with lower-semicontinuous payoffs, Mathematics of Operations Research 35 (4) (2010) 742-755.

[8] R. Purves, W. Sudderth, Perfect information games with upper semicontinuous payoffs, Mathematics of Operations Research 36 (3) (2011) 468-473.

[9] R. Bloem, K. Chatterjee, T. A. Henzinger, B. Jobstmann, Better quality in synthesis through quantitative objectives, in: A. Bouajjani, O. Maler (Eds.), Computer Aided Verification, Vol. 5643 of Lecture Notes in Computer Science, Springer Berlin Heidelberg, 2009, pp. 140-156. doi:10. 1007/978-3-642-02658-4\_14.

[10] M. Ummels, D. Wojtczak, The complexity of Nash equilibria in limitaverage games, in: J.-P. Katoen, B. König (Eds.), CONCUR 2011 Concurrency Theory, Springer, Berlin, Heidelberg, 2011, pp. 482-496. doi:10.1007/978-3-642-23217-6\_32.

[11] R. Selten, Spieltheoretische Behandlung eines Oligopolmodells mit Nachfrageträgheit, Z. Gesamte Staatswissenschaft 12 (1965) 301-324.

[12] S. Le Roux, Generalisation and formalisation in game theory, Ph.D. thesis, Ecole Normale Supérieure de Lyon (January 2008).

[13] T. Roughgarden, E. Tardos, Introduction to the inefficiency of equilibria, in: 41], Cambridge University Press, 2007, pp. 443-459.

[14] K. Chatterjee, T. A. Henzinger, M. Jurdziński, Games with secure equilibria, Theoretical Computer Science 365 (1-2) (2006) 67-82.

[15] T. Brihaye, V. Bruyère, J. De Pril, Equilibria in quantitative reachability games, in: Proc. of CSR, Vol. 6072 of LNCS, Springer, 2010. doi:10. 1007/978-3-642-13182-0\_7.

[16] T. Brihaye, V. Bruyère, J. De Pril, H. Gimbert, On (subgame perfect) secure equilibrium in quantitative reachability games, Logical Methods in Computer Science 9. 
[17] D. A. Martin, An extension of Borel determinacy, Annals of Pure and Applied Logic 49 (3) (1990) 279 - 293. doi:10.1016/0168-0072(90)90029-2.

[18] D. Fudenberg, D. Levine, Subgame-perfect equilibria of finite- and infinitehorizon games, Journal of Economic Theory 31 (2) (1983) 251 - 268. doi: http://dx.doi.org/10.1016/0022-0531(83)90076-5.

[19] C. Alós-Ferrer, K. Ritzberger, Equilibrium existence for large perfect information games, Journal of Mathematical Economics 62 (2016) 5 - 18. doi:j.jmateco.2015.10.005

घURL http://www.sciencedirect.com/science/article/pii/ S0304406815001238

[20] W. He, Y. Sun, Dynamic games with almost perfect information, arXiv:1503.08900 (2015).

[21] J.-F. Mertens, S. Sorin, S. Zamir, Repeated games, Cambridge University Press, 2015.

[22] A. Mashiah-Yaakovi, Correlated equilibria in stochastic games with Borel measurable payoffs, Dynamic Games and Applications 5 (1) (2015) 120135. doi:10.1007/s13235-014-0122-2.

URL https://doi .org/10.1007/s13235-014-0122-2

[23] E. Solan, N. Vieille, Deterministic multi-player Dynkin games, Journal of Mathematical Economics 39 (8) (2003) 911 - 929. doi:10.1016/ S0304-4068(03)00021-1.

[24] D. A. Martin, The determinacy of Blackwell games, Journal of Symbolic Logic 63 (4) (1998) 1565-1581. doi:10.2307/2586667.

[25] T. Krieger, On Pareto equilibria in vector-valued extensive form games, Mathematical Methods of Operations Research 58 (2003) 449-458.

[26] S. Le Roux, Acyclic preferences and existence of sequential Nash equilibria: a formal and constructive equivalence, in: TPHOLs, International Conference on Theorem Proving in Higher Order Logics, Lecture 
Notes in Computer Science, Springer, 2009, pp. 293-309. doi:10.1007/ 978-3-642-03359-9\_21.

[27] J. F. Nash, Equilibrium Points in N-Person Games, Proceedings of the National Academy of Sciences 36 (1) (1950) 48-49. arXiv:http://www. pnas.org/content/36/1/48.full.pdf+html. URL http: //www . pnas . org/content/36/1/48. short

[28] D. Bridges, First steps in constructive game theory, Mathematical Logic Quaterly 50 (2004) 501-506.

[29] A. Pauly, How incomputable is finding Nash equilibria?, Journal of Universal Computer Science 16 (18) (2010) 2686-2710. doi:10.3217/ jucs-016-18-2686.

[30] S. Le Roux, A. Pauly, Weihrauch degrees of finding equilibria in sequential games, in: A. Beckmann, V. Mitrana, M. Soskova (Eds.), Evolving Computability, Vol. 9136 of Lecture Notes in Computer Science, Springer, 2015, pp. 246-257. doi:10.1007/978-3-319-20028-6\_25.

[31] A. Pauly, Computability on the countable ordinals and the HausdorffKuratowski theorem (extended abstract), in: G. F. Italiano, G. Pighizzini, D. T. Sannella (Eds.), Mathematical Foundations of Computer Science 2015, Vol. 9234 of Lecture Notes in Computer Science, Springer, 2015, pp. 407-418. doi:10.1007/978-3-662-48057-1\_32. URL http://dx.doi .org/10.1007/978-3-662-48057-1_32

[32] S. Le Roux, Infinite subgame perfect equilibrium in the Hausdorff difference hierarchy, in: T. M. Hajiaghayi, R. M. Mousavi (Eds.), TTCS 2015, Revised Selected Papers, Springer, 2016, pp. 147-163, also available at http://arxiv.org/abs/1505.06320. doi:10.1007/978-3-319-28678-5 -11.

[33] V. Bruyère, S. Le Roux, A. Pauly, J.-F. Raskin, On the existence of weak subgame perfect equilibria, in: J. Esparza, A. S. Murawski (Eds.), 
Foundations of Software Science and Computation Structures (FOSSACS 978-3-662-54458-7\_9

URL http://dx.doi.org/10.1007/978-3-662-54458-7_9

[34] T. Brihaye, G. Geeraerts, M. Hallet, S. L. Roux, Dynamics and coalitions in sequential games, in: Proceedings Eighth International Symposium on Games, Automata, Logics and Formal Verification, GandALF 2017, 2017, pp. 136-150. doi:10.4204/EPTCS.256.10. URL https://doi.org/10.4204/EPTCS.256.10

[35] R. J. Aumann, Contributions to the Theory of Games, Princeton University Press, 1959, Ch. Acceptable Points in General Cooperative n-Person Games. doi:10.1515/9781400882168-018.

[36] R. Nessah, G. Tian, On the existence of strong nash equilibria, Journal of Mathematical Analysis and Applications 414 (2) (2014) 871 - 885. doi:j.jmaa.2014.01.030. URL http://www.sciencedirect.com/science/article/pii/ S0022247X14000377

[37] V. Bruyère, N. Meunier, J.-F. Raskin, Secure equilibria in weighted games, in: Proc. of CSL/LICS, CSL-LICS '14, ACM, 2014, pp. 26:1-26:26. doi: $10.1145 / 2603088.2603109$.

URL http://doi.acm.org/10.1145/2603088.2603109

[38] J. De Pril, J. Flesch, J. Kuipers, G. Schoenmakers, K. Vrieze, Existence of secure equilibrium in multi-player games with perfect information, in: E. Csuhaj-Varjú, M. Dietzfelbinger, Z. Ésik (Eds.), Mathematical Foundations of Computer Science 2014, Springer Berlin Heidelberg, Berlin, Heidelberg, 2014, pp. 213-225. doi:10.1007/978-3-662-44465-8\_19.

[39] J. F. Mertens, Repeated games, in: Proc. Internat. Congress Mathematicians, American Mathematical Society, 1987, pp. 1528-1577. 
[40] J. Flesch, A. Predtetchinski, A characterization of subgame-perfect equilibrium plays in Borel games of perfect information, Mathematics of Operations Researchdoi:10.1287/moor.2016.0843.

[41] N. Nisan, T. Roughgarden, E. Tardos, V. Vazirani (Eds.), Algorithmic Game Theory, Cambridge University Press, 2007. 